\title{
Quantum-Enabled Communication without a Phase Reference
}

\author{
Quntao Zhuang®* \\ Department of Electrical and Computer Engineering and James C. Wyant College of Optical Sciences, \\ University of Arizona, Tucson, Arizona 85721, USA
}

(Received 22 October 2020; accepted 14 January 2021; published 10 February 2021)

\begin{abstract}
A phase reference has been a standard requirement in continuous-variable quantum sensing and communication protocols. However, maintaining a phase reference is challenging due to environmental fluctuations, preventing quantum phenomena such as entanglement and coherence from being utilized in many scenarios. We show that quantum communication and entanglement-assisted communication without a phase reference are possible, when a short-time memory effect is present. The degradation in the communication rate of classical or quantum information transmission decreases inversely with the correlation time. Exact solutions of the quantum capacity and entanglement-assisted classical and quantum capacity for pure dephasing channels are derived, where non-Gaussian multipartite-entangled states show strict advantages over usual Gaussian sources. For thermal-loss dephasing channels, lower bounds of the capacities are derived. The lower bounds also extend to scenarios with fading effects in the channel. In addition, for entanglement-assisted communication, the lower bounds can be achieved by a simple phaseencoding scheme on two-mode squeezed vacuum sources, when the noise is large.
\end{abstract}

DOI: 10.1103/PhysRevLett.126.060502

Quantum physics has reshaped our understanding of communication. The Shannon capacity has been generalized to the Holevo-Schumacher-Westmoreland (HSW) classical capacity [1-3] to incorporate quantum effects during transmission. Entanglement has also enabled nonclassical phenomena in communication, such as superadditivity [4-9] and capacity boost from entanglement assistance (EA) [10-18]. Moreover, reliable transmission of quantum information is possible, established by the Lloyd-Shor-Devetak quantum capacity theorem [19-21].

Apart from the information theoretical advances, the physical realization of quantum-enabled communication protocols, inevitably in the optical domain, have been extensively studied. Although the classical capacity has been found additive [22], the quantum capacity of noisy optical communication is still an open question [23-26]. In EA communication, the advantage of entanglement has been known for decades and, surprisingly, thrives even more in the presence of loss and noise [12]. More recently, practical EA classical communication protocols have been designed [27] and experimentally demonstrated to beat the HSW capacity [28].

Despite the recent progresses, the need of phase stabilization - the maintenance of a phase reference between the

Published by the American Physical Society under the terms of the Creative Commons Attribution 4.0 International license. Further distribution of this work must maintain attribution to the author(s) and the published article's title, journal citation, and DOI. sender and the receiver-in the above protocols places a serious constraint on their applicability. Even in a wellcontrolled experimental condition [28], the instability of phase-locking limits the time duration of EA communication. In the presence of environmental fluctuations, phase stabilization over real communication links requires a highly nontrivial feedback control system [29], which might be impossible for wireless scenarios.

In this Letter, surprisingly, we show that quantum-enabled communication without a phase reference is possible and advantageous. We adopt the non-Gaussian memory channel model in Ref. [30], where phase fluctuations have a finite timescale such that at most $m$ signal modes can be sent out with a fixed unknown random phase. Despite facing challenges brought by the non-Gaussian nature of the channel, we exactly solve the quantum capacity, EA classical capacity and EA quantum capacity of such a bosonic dephasing channel. In the EA case, optimal encoding requires a non-Gaussian multipartite-entangled state and is strictly better than the conventional Gaussian encoding known to be optimal in the absence of phase noise.

With thermal-loss effects in play, we provide lower and upper bounds of the communication rates, showing that phase noise only decreases the capacity by at most a constant $\sim \log _{2}(m) / m$ bits per mode. In addition, when the thermal noise in the communication link is high, we show that phase encoding on a two-mode squeezed vacuum (TMSV) achieves the EA capacity lower bounds, assuming optimum receivers for decoding. Extending the bounds to channel fading scenarios [31-33], we show that the EA advantages persist. 


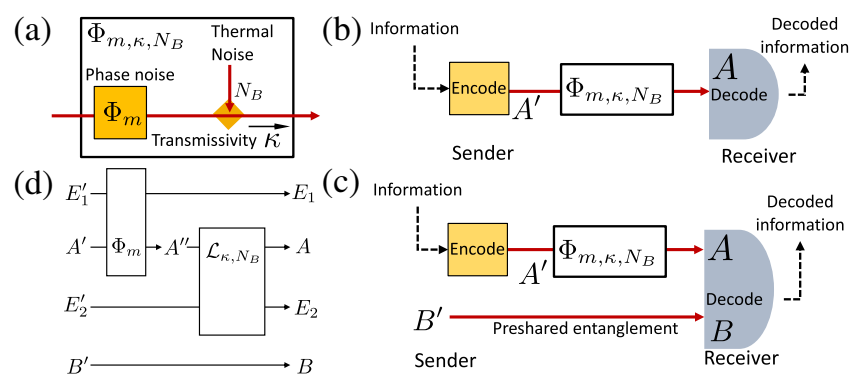

FIG. 1. (a) Schematic of the overall thermal-loss dephasing channel $\Phi_{m, \kappa, N_{B}}$, which is composed of a pure dephasing channel $\Phi_{m}$ and a bosonic thermal-loss channel with transmissivity $\kappa$ and noise $N_{B}$. (b) Schematic of a communication protocol. (c) Schematic of an EA communication protocol. (d) Channel diagram to assist the information-theoretical analyses. Stinespring dilations are shown for both channels, with environment $E_{1}^{\prime}$ and $E_{2}^{\prime}$.

A channel model of phase noise.-Light propagation in a lossy noisy media is typically modeled by a phasecovariant bosonic thermal-loss channel $\mathcal{L}_{\kappa, N_{B}}$ [22,34] described by the beam-splitter transformation $\hat{a} \rightarrow$ $\sqrt{\kappa} \hat{a}+\sqrt{1-\kappa} \hat{e}$, on the input annihilation operator $\hat{a}$, with $\hat{e}$ in a thermal state of mean photon number $N_{B} /(1-\kappa)$.

In addition to the noise and loss, the quantum state of light propagation also picks up a phase. In a bosonic dephasing channel $\Phi_{m}$ introduced by Ref. [30], the $m$-mode input state $\hat{\sigma}$ experiences an identical but fully random phase, leading to the output

$\Phi_{m}(\hat{\sigma})=\left\langle\hat{U}_{\theta} \hat{\sigma} \hat{U}_{\theta}^{\dagger}\right\rangle_{\theta} \equiv \frac{1}{2 \pi} \int_{0}^{2 \pi} d \theta \hat{U}_{\theta} \hat{\sigma} \hat{U}_{\theta}^{\dagger}=\sum_{n=0}^{\infty} p_{n} \hat{\sigma}_{n}$,

where $\hat{U}_{\theta}=e^{i \theta \hat{n}}$ and $\hat{n}$ is the total photon number operator. The channel effectively projects the input to subspaces, each with a different fixed total photon number $n$, through the projectors $\hat{\Pi}_{n}=\sum_{|\boldsymbol{n}|=n}|\boldsymbol{n}\rangle\langle\boldsymbol{n}|$, where $\boldsymbol{n}=\left(n_{1}, \ldots, n_{m}\right)$ is an $m$-dimensional vector representing the photon number in each mode, and we denote $|\boldsymbol{n}|=\sum_{k=1}^{m} n_{k}$. Therefore, we have the final expression in Eq. (1) with $p_{n}=\operatorname{tr}\left(\hat{\Pi}_{n} \hat{\sigma}\right)$ and $\hat{\sigma}_{n}=\hat{\Pi}_{n} \hat{\sigma} \hat{\Pi}_{n} / p_{n}$. For later use, we introduce the complementary channel $\Phi_{m}^{c}(\hat{\sigma})=\sum_{k=0}^{\infty} \sum_{|\boldsymbol{n}|=k}\langle\boldsymbol{n}|\hat{\sigma}| \boldsymbol{n}\rangle|k\rangle\langle k|$, which produces the environment $E_{1}$ in a Stinespring dilation [see Fig. 1(d)]. As $\Phi_{m}^{c}$ is entanglement breaking [35], $\Phi_{m}$ is a Hadamard channel [36] and its entire capacity region [15,37,38] is additive [39].

Combining the above, the light propagation process can be modeled as an overall thermal-loss dephasing channel

$$
\Phi_{m, \kappa, N_{B}}=\mathcal{L}_{\kappa, N_{B}}^{\otimes m} \circ \Phi_{m}=\Phi_{m} \circ \mathcal{L}_{\kappa, N_{B}}^{\otimes m},
$$

as shown in Fig. 1(a), where $\mathcal{L}_{\kappa, N_{B}}^{\otimes m}$ commutes with $\Phi_{m}$.

Capacity formula.-In a general communication scenario [see Figs. 1(b) and 1(c)], the sender encodes the quantum or classical information into the signal input $A^{\prime}$ and sends the signal through the media, e.g., a fiber or open-space link. Upon receiving the signal $A$, the receiver decodes the classical or quantum information. In an EA communication scenario, in addition to the above, the sender and receiver preshare entanglement between the input $A^{\prime}$ and an idler $B^{\prime}$, potentially through a quantum network. The idler is stored in the receiver's quantum memory for later assistance in the decoding. To facilitate the information-theoretical analysis, we introduce the diagram of channels in Fig. 1(d), where the channels are shown as Stinespring dilations acting on environment $E_{1}^{\prime}$, $E_{2}^{\prime}$ and the inputs.

First, we start with the transmission of quantum information, without entanglement assistance. The ultimate rate of quantum communication over a general quantum channel $\Phi$ is given by a regularized maximization

$$
Q(\Phi)=\lim _{N \rightarrow \infty} \frac{1}{N} \max _{\hat{\sigma}} J\left(\hat{\sigma}, \Phi^{\otimes N}\right),
$$

over the input state $\hat{\sigma}$ of $A^{\prime}$, where the coherent information for a single channel use $J(\hat{\sigma}, \Phi)=S[\Phi(\hat{\sigma})]-S\left[\Phi^{c}(\hat{\sigma})\right]=$ $S\left(\hat{\rho}_{A}\right)-S\left(\hat{\rho}_{E_{1} E_{2}}\right)$, as shown in Fig. 1(d). Here $\hat{\rho}$ is the output state and the von Neumann entropy $S(\hat{\delta}) \equiv-\operatorname{tr}\left(\hat{\delta} \log _{2} \hat{\delta}\right)$ for state $\hat{\delta}$. As the input Hilbert space dimension is infinite, we adopt the widely used $[12,27,30,34]$ mean photon number constraint $m E$ per channel used during the entire $N \rightarrow \infty$ channel uses, such that the capacity is finite.

Suppose one has preshared entanglement assistance, the rate of quantum information transmission is increased to a maximization of the quantum mutual information between the received signal $A$ and the idler $B$ [12]

$$
Q_{\mathrm{EA}}(\Phi)=\frac{1}{2} C_{\mathrm{EA}}(\Phi)=\frac{1}{2} \max _{\hat{\phi}} I(A: B)_{\hat{\rho}},
$$

over the input state $\hat{\phi}$ of $A^{\prime}$ and $B^{\prime}$. In the first equality, we utilize the fact that the EA quantum capacity is precisely half of the EA classical capacity, via the reduction from teleportation [40] and superdense coding [10]. As shown in Fig. 1(d), the quantum mutual information $I(A: B)_{\hat{\rho}}=S\left(\hat{\rho}_{A}\right)+S\left(\hat{\rho}_{B}\right)-S\left(\hat{\rho}_{E_{1} E_{2}}\right)$, where we utilized $S\left(\hat{\rho}_{A B}\right)=S\left(\hat{\rho}_{E_{1} E_{2}}\right)$, due to the purity of $A B E_{1} E_{2}$.

When a phase reference is present, the EA capacity $C_{\mathrm{EA}}\left(\mathcal{L}_{\kappa, N_{B}}\right)$ of the thermal-loss channel $\mathcal{L}_{\kappa, N_{B}}$ is exactly solvable and known to be achieved by the TMSV encoding $[12,18,41,42]$, thanks to the Gaussian nature of the channel; similarly, the HSW capacity [22] $C\left(\mathcal{L}_{\kappa, N_{B}}\right)$ can be achieved by a Gaussian ensemble of coherent states (see Supplemental Material [43]). On the other hand, even with a phase reference present, only upper and lower bounds of the quantum capacity $Q\left(\mathcal{L}_{\kappa, N_{B}}\right)$ are known [23-26]; except in the noiseless case $N_{B}=0$, the exact solution is known and achieved by a thermal state. 
Exact solution for a pure dephasing channel.-We begin our analyses with a pure dephasing channel in the absence of additional noise $\left(N_{B}=0, \kappa=1\right)$. The HSW classical capacity $C\left(\Phi_{m}\right) / m=g(E)$ can be solved [30], where $g(n)=(n+1) \log _{2}(n+1)-n \log _{2} n$ is the entropy of a thermal state with mean photon number $n$. Extending to the quantum-enabled case is much more involved due to the complication from entanglement adding to the nonGaussian nature of the problem; our first result is the exact solutions of the quantum capacity and EA capacity of the pure dephasing channel $\Phi_{m}$. As $\Phi_{m}$ is projective in the total photon number bases and Hadamard, we can reduce the optimization in Eqs. (3) and (4) to an optimization over the $m$-mode photon number distribution $P_{n}$, leading to [43]

$$
\begin{aligned}
Q\left(\Phi_{m}\right)=\max _{\left\{P_{n}\right\}}\left[H\left(\left\{P_{n}\right\}_{n}\right)-H\left(\left\{P_{n}^{t}\right\}_{n}\right)\right], \\
C_{\mathrm{EA}}=2 Q_{\mathrm{EA}}=\max _{\left\{P_{n}\right\}}\left[2 H\left(\left\{P_{\boldsymbol{n}}\right\}_{n}\right)-H\left(\left\{P_{n}^{t}\right\}_{n}\right)\right],
\end{aligned}
$$

under the energy constraint $\sum_{n} n P_{n}^{t}=m E$, with the total photon number distribution $P_{n}^{t}=\sum_{|n|=n} P_{n}$. For $m=1$, we can immediately see that $Q\left(\Phi_{m}\right)=0$ and $C_{\mathrm{EA}}\left(\Phi_{m}\right)=$ $C\left(\Phi_{m}\right)$ due to $P_{n}^{t}=P_{|n|}$; therefore, quantum advantage in the absence of phase reference is impossible for the single-mode case. As a thermal state maximizes the von Neumann entropy given the energy constraint, we can directly obtain an upper bound as $Q\left(\Phi_{m}\right) \leq m g(E)$ and $C_{\mathrm{EA}}\left(\Phi_{m}\right) \leq 2 m g(E)=2 C\left(\Phi_{m}\right)$.

The optimization in Eqs. (5) can be solved exactly. Noticing that the constraint only affects $P_{n}^{t}$, and that $P_{n}$ only appears in the first entropy term, it is optimal to distribute $P_{n}^{t}$ equally between $P_{n}$ with identical $|\boldsymbol{n}|=n$. Then, utilizing Lagrange multipliers, one obtains the optimal $m$-mode input

$$
\hat{\sigma}_{A^{\prime}}=\sum_{|\boldsymbol{n}|=m E}\left(C_{|\boldsymbol{n}|+m-1}^{m-1}\right)^{-1}|\boldsymbol{n}\rangle\left\langle\left.\boldsymbol{n}\right|_{A^{\prime}}\right.
$$

up to integer rounding [43] for the quantum capacity, and the optimal $2 m$-mode input state [43]

$$
\hat{\phi}_{A^{\prime} B^{\prime}} \propto \sum_{\boldsymbol{n}} \sqrt{C_{|\boldsymbol{n}|+m-1}^{m-1} \tilde{\lambda}_{1}^{|\boldsymbol{n}|}}|\boldsymbol{n}\rangle_{A^{\prime}}|\boldsymbol{n}\rangle_{B^{\prime}}
$$

for EA communication, where $\tilde{\lambda}_{1}$ is determined by the energy constraint and $C_{a}^{b}=(a !) /[b !(a-b) !]$ is the binomial coefficient. The corresponding $Q\left(\Phi_{m}\right)=\log _{2}\left(C_{m E+m-1}^{m-1}\right)$ up to integer rounding [43], and $C_{\mathrm{EA}}=2 Q_{\mathrm{EA}}$ can be evaluated efficiently from Eq. (5b) with the above input state. Interestingly, as $|\boldsymbol{n}|=\sum_{k=1}^{m} n_{k}$, the prefactor $C_{|\boldsymbol{n}|+m-1}^{m-1}$ prevents $P_{\boldsymbol{n}}$ to be written as any product of distributions over each variable $n_{k}$, making the optimal input $\hat{\phi}_{A^{\prime} B^{\prime}}$ a
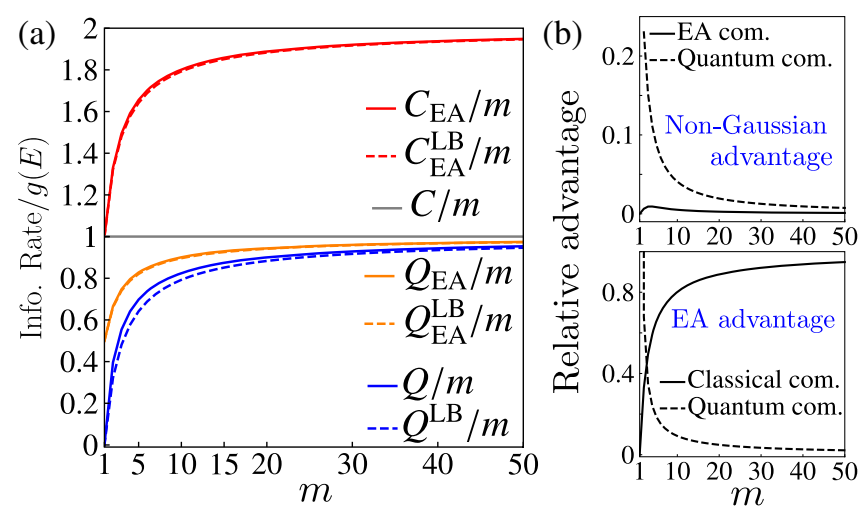

FIG. 2. (a) Ratio of the information rate over the classical capacity per mode $C=g(E)$ (gray solid) for a pure dephasing channel $\Phi_{m}$, with an input energy per mode $E=1$ : EA classical capacity per mode $C_{\mathrm{EA}} / m$ (red solid), EA quantum capacity per mode $Q_{\mathrm{EA}} / m$ (orange solid), and quantum capacity per mode $Q / m$ (blue solid). The dashed lines with the corresponding color are the lower bound offered by Gaussian-encoding schemes. (b) Relative advantages in information rate for non-Gaussian encoding (top) and entanglement assistance (bottom).

non-Gaussian 2m-mode-entangled state (see Supplemental Material [43]).

As we see in Fig. 2(a), the quantum capacity (blue solid) increases from zero quickly as the memory $m$ increases; at the same time, entanglement provides advantages in both quantum communication (orange solid) and classical communication (red solid), as emphasized in the bottom panel of Fig. 2(b). It is worthy to note that these capacityachieving inputs provide strict advantages over suboptimal Gaussian inputs, which are optimal in the presence of a phase reference. As a consequence, the thermal input provides a lower bound (LB) for the quantum capacity $Q^{\mathrm{LB}}$, while an independent and identical (IID) product of TMSV states provides lower bounds for EA capacities $C_{\mathrm{EA}}^{\mathrm{LB}}$ and $Q_{\mathrm{EA}}^{\mathrm{LB}}$, as shown in dashed lines in Fig. 2(a). The advantage is larger in the quantum communication case, up to $20 \%$; whereas it is smaller for the EA communication, up to $1 \%$, as shown in the top panel of Fig. 2(b).

Capacity bounds for thermal-loss dephasing.-The exact evaluation of the capacities for $\Phi_{m, \kappa, N_{B}}$ in the presence of loss and noise is challenging [43]. Instead, we obtain upper and lower bounds. Combining the data-processing inequality (bottleneck inequality) and the upper bound (UB) in Refs. [24,25], we can obtain an upper bound

$Q^{(\mathrm{UB})}\left(\Phi_{m, \kappa, N_{B}}\right) \equiv \min \left[Q\left(\Phi_{m}\right), m \min _{1 \leq G_{1} \leq 1+N_{B}} f\left(G_{1}\right)\right]$,

where $Q\left(\Phi_{m}\right)$ is given by the exact solution and

$$
f\left(G_{1}\right)=\max \left\{g\left(\bar{\eta} E^{\prime}\right)-g\left[(1-\bar{\eta}) E^{\prime}\right], 0\right\}
$$


is derived for upper bounding $Q\left(\mathcal{L}_{\kappa, N_{B}}\right)$. Here the constants $E^{\prime}=G_{2} E+\left(G_{2}-1\right), \bar{\eta}=1-\left(N_{B}+1-\eta\right) / G_{1}$, and $G_{2}=\eta /\left[G_{1}-\left(N_{B}+1-\eta\right)\right]$. Similarly, for the EA capacity we have $C_{\mathrm{EA}}\left(\Phi_{m, \kappa, N_{B}}\right) \leq m C_{\mathrm{EA}}\left(\mathcal{L}_{\kappa, N_{B}}\right)$ is upper bounded by the EA capacity of the thermal-loss channel.

On the other hand, we obtain the lower bounds

$$
\begin{aligned}
J\left(\hat{\rho}, \Phi_{m, \kappa, N_{B}}\right) & \geq S\left(\hat{\rho}_{A}\right)-S\left(\hat{\rho}_{E_{1}}\right)-S\left(\hat{\rho}_{E_{2}}\right), \\
I(A: B)_{\hat{\rho}} & \geq S\left(\hat{\rho}_{A}\right)+S\left(\hat{\rho}_{B}\right)-S\left(\hat{\rho}_{E_{1}}\right)-S\left(\hat{\rho}_{E_{2}}\right),
\end{aligned}
$$

from the subadditivity of entropy $S\left(\hat{\rho}_{E_{1} E_{2}}\right) \leq S\left(\hat{\rho}_{E_{1}}\right)+$ $S\left(\hat{\rho}_{E_{2}}\right)$. In general, there will be correlations between $E_{1}$ and $E_{2}$ and the above bounds are not tight. The above inequality provides ways to circumvent the non-Gaussian nature of the channel as we explain below.

Consider Fig. 1(d): suppose we input a photon number diagonal state as $A^{\prime}$; we have $A^{\prime \prime}$ in a state identical to the input $A^{\prime}$. Moreover, the reduced state of the environment mode $E_{2}$ and that of output $A, B$ are identical to the states in a scenario without the channel $\Phi_{m}$. Now suppose the inputs are Gaussian, the only non-Gaussian part $E_{1}$ involved in Inequalities (10) can also be efficiently calculated from the total photon number distribution. Therefore, considering IID thermal states $\hat{\rho}_{\mathrm{th}}^{\otimes m}$ for the coherent information and IID TMSVs for the EA capacity, from Inequalities (10) we have the lower bounds

$$
\begin{aligned}
& Q^{\mathrm{LB}} \equiv J\left(\hat{\rho}_{\mathrm{th}}^{\otimes m}, \mathcal{L}_{\kappa, N_{B}}^{\otimes m}\right)-H\left(\left\{P_{n}^{t}\right\}\right), \\
& C_{\mathrm{EA}}^{\mathrm{LB}} \equiv 2 Q_{\mathrm{EA}}^{\mathrm{LB}} \equiv m C_{\mathrm{EA}}\left(\mathcal{L}_{\kappa, N_{B}}\right)-H\left(\left\{P_{n}^{t}\right\}\right),
\end{aligned}
$$

where both the coherent information $J\left(\hat{\rho}_{\mathrm{th}}^{\otimes m}, \mathcal{L}_{\kappa, N_{B}}^{\otimes m}\right)$ and the EA capacity $C_{\mathrm{EA}}\left(\mathcal{L}_{\kappa, N_{B}}\right)$ have closed-form solutions (see Supplemental Material [43]); the Shannon entropy $H(\cdot)$ is over distribution

$$
P_{n}^{t}=C_{n+m-1}^{m-1} \frac{E^{n}}{(E+1)^{n+m}} .
$$

Although not having a closed form, it can be efficiently evaluated numerically. Furthermore, for $m \gg 1$, from the law of large numbers, $P_{n}^{t}$ approaches a Gaussian distribution with mean $m E$ and variance $m E(E+1)$; therefore, asymptotically $H\left(\left\{P_{n}^{t}\right\}\right) \simeq \log _{2}(\epsilon \sqrt{m E(E+1)})$, where $\epsilon=\sqrt{2 \pi e} \simeq 4.13$ is a constant. In most of the parameter region, we indeed see a good agreement between the above asymptotic expressions and exact results [43]. An important take-away of Inequalities (11) is that the degradation caused by the phase noise is only of the order of $\log _{2}(m) / m$ bits per mode.

We consider our upper and lower bounds in two typical examples. Figure 3(a) represents a microwave region, where background noise $N_{B} \sim 1$ and transmissivity

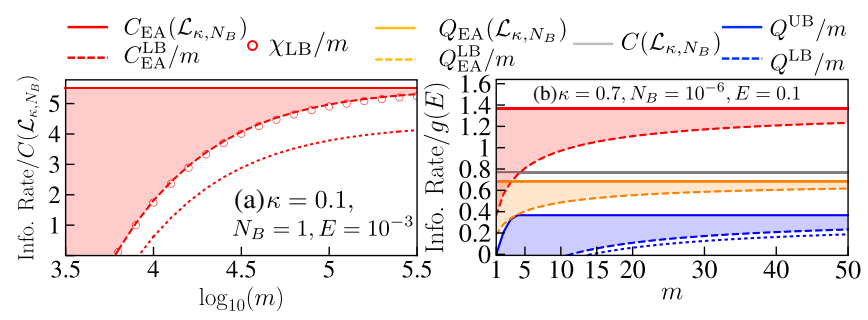

FIG. 3. Information rate ratio. The EA classical capacity (red), EA quantum capacity (orange), and quantum capacity (blue) lies in the colored region. Upper bounds are in solid colored lines and lower bounds in dashed colored lines. (a) Ratio of information rate over the classical capacity $C\left(\mathcal{L}_{\kappa, N_{B}}\right)$ of a thermal-loss channel, with an input power $E=10^{-3}$ per mode and the channel transmissivity fixed at $\kappa=0.1$ and noise $N_{B}=1 . \chi_{\mathrm{LB}} / m$ (red circles) is the lower bound of the accessible information of phase encoding. (b) Ratio of information rate over $g(E)$, with an input power $E=0.1$ per mode and $\kappa=0.7, N_{B}=10^{-6}$. The classical capacity of a thermal-loss channel $C\left(\mathcal{L}_{\bar{\kappa}, N_{B}}\right)$ is also plotted with gray solid line for comparison. The dotted red line in (a) is the lower bound with Rayleigh fading of $\bar{\kappa}=0.1$ for EA classical capacity, and the dotted blue line in (b) is the lower bound for quantum capacity with flat fading in $\kappa \in[0.4,1]$.

$\kappa=0.1$ (note that larger $N_{B}$ provides similar results [43]). Figure 3(b) represents an optical fiber connection or near-field free-space link, where $N_{B} \sim 10^{-6}$ [55]. In these plots, the upper bounds are plotted with solid lines, while lower bounds are dashed lines of the same color. The colored region indicates where the true capacity lies. In the noisy case, we do not know the exact classical capacity of $\Phi_{m, \kappa, N_{B}}$, so we take the ratio of the information rate over $C\left(\mathcal{L}_{\kappa, N_{B}}\right) \geq C\left(\Phi_{m, \kappa, N_{B}}\right) / m$ in Fig. 3(a). Similar to the noiseless case, the lower bound converges to the upper bound $C_{\mathrm{EA}}\left(\mathcal{L}_{\kappa, N_{B}}\right)$ quickly and revives the huge advantage over the HSW classical capacity when noise $N_{B}$ is large $[12,27]$. The number of modes for saturation $m \sim 10^{5}$ in the microwave region, which corresponds to less than a millisecond for a gigahertz bandwidth microwave source. While for the optical case of Fig. 3(b), the saturation is even faster, and we see advantages both in classical communication (red vs gray) and quantum communication (orange vs blue) at very small $m$.

Encoding achieving the EA lower bound.-With upper and lower bounds in hand, we now proceed to investigate practical encoding schemes for EA communication in the presence of phase noise. Below, we will focus on the EA classical communication; EA quantum communication can be completed with the same protocol plus teleportation. We consider the performance of independent phase encoding on an IID product of TMSVs - applying a phase rotation $\hat{U}_{\theta}$ with uniform $\theta \in[0,2 \pi)$ on each signal mode, which has been shown to saturate the EA capacity in the absence of phase noise [27]. Combing the optimality results in Ref. [27], we can use the same technique in deriving the capacity lower bounds to obtain the accessible (Holevo) information [43] 


$$
\frac{1}{m} \chi_{\mathrm{LB}}=C_{\mathrm{EA}}\left(\mathcal{L}_{\kappa, N_{B}}\right)-\frac{1}{m} H\left(\left\{P_{n}^{t}\right\}\right)+O\left(1 / N_{B}^{2}\right),
$$

which achieves the capacity lower bound in the $N_{B} \gg 1$ limit. We verify the above conclusions numerically in Fig. 3(a). When $N_{B}=1$ the accessible information lower bound per mode $\chi_{\mathrm{LB}} / m$ (red open circles) overlaps with the EA capacity lower bound (red dashed).

Extension to fading channels.-For dynamic links such as wireless links to mobile devices [56], environmental fluctuations can affect more than just the phase, but also cause the transmissivity to vary with time; therefore, the overall channel output on the $m$-mode input $\hat{\sigma}$

$$
\mathcal{R}_{m, \bar{\kappa}, N_{B}}(\hat{\sigma})=\left\langle\Phi_{m, x^{2}, N_{B}}(\hat{\sigma})\right\rangle_{f(x)},
$$

where $f(x)$ can be any type of fading distribution in $[0,1]$ with an average power transmissivity $\bar{\kappa}$. We can also obtain efficiently calculable lower bounds of the capacities of $\mathcal{R}_{m, \bar{\kappa}, N_{B}}$ as [43]

$$
\begin{aligned}
\frac{1}{m} C_{\mathrm{EA}}^{\mathrm{LB}} \equiv & \frac{2}{m} Q_{\mathrm{EA}}^{\mathrm{LB}} \equiv \frac{1}{m} Q^{\mathrm{LB}}+g(E) \equiv\left\langle g\left(x^{2} E+N_{B}\right)\right\rangle_{f(x)} \\
& +g(E)-g\left(\nu_{+}\right)-g\left(\nu_{-}\right)-H\left(\left\{P_{n}^{t}\right\}\right) / m,
\end{aligned}
$$

where $\nu_{ \pm}=\left(\sqrt{(A+S)^{2}-4 C^{2}} \pm(S-A)-2\right) / 4$, with the constants $A \equiv 2\left(\bar{\kappa} E+N_{B}\right)+1, S \equiv 2 E+1$, and $C \equiv$ $2\langle x\rangle_{f(x)} \sqrt{E(E+1)}$.

For the EA communication scenario, as the transmissivity is typically low, we adopt the Rayleigh distribution [31-33] $f(x) \sim x \exp \left(-x^{2} / \bar{\kappa}\right)$ with proper truncation in $[0,1]$. For quantum communication, the capacity is sharply zero when $\kappa<0.5$; therefore, it is more reasonable to consider a flat fading of $x^{2} \in[\bar{\kappa}-\delta \kappa, \bar{\kappa}+\delta \kappa]$. We evaluate the lower bounds in Fig. 3 as the dotted lines for the same average transmissivity in each case. We see the quantum capacity is only mildly decreased due to fading [dotted blue line in Fig. 3(b)], while the lower bound is decreased appreciably by fading for EA communication [dotted red line in Fig. 3(a)]; however, the scaling of the advantage over the HSW classical capacity survives (see Supplemental Material [43]).

Discussion.-In this Letter, we show that quantum advantages in communication are possible without a phase reference, assuming a finite-time memory effect in the channel. We exactly solve the EA capacities and quantum capacity of a pure dephasing channel, showing that the optimal input of a non-Gaussian multipartite-entangled state is strictly better than the Gaussian-entangled source. In the presence of additional thermal noise, loss, and fading effects, we derive upper and lower bounds of the capacities, which shows that the degradation from the absence of a phase reference is mild. Many future directions can be explored, including an extension to cases with finite phase noise [57] and practical protocol design for achieving the lower bounds.
Q.Z. acknowledges the Defense Advanced Research Projects Agency (DARPA) under Young Faculty Award (YFA) Grant No. N660012014029 and Craig M. Berge Dean's Faculty Fellowship of University of Arizona.

*zhuangquntao@email.arizona.edu

[1] P. Hausladen, R. Jozsa, B. Schumacher, M. Westmoreland, and W. K. Wootters, Classical information capacity of a quantum channel, Phys. Rev. A 54, 1869 (1996).

[2] B. Schumacher and M. D. Westmoreland, Sending classical information via noisy quantum channels, Phys. Rev. A 56, 131 (1997).

[3] A. S. Holevo, The capacity of the quantum channel with general signal states, IEEE Trans. Inf. Theory 44, 269 (1998).

[4] M. B. Hastings, Superadditivity of communication capacity using entangled inputs, Nat. Phys. 5, 255 (2009).

[5] G. Smith and J. Yard, Quantum communication with zerocapacity channels, Science 321, 1812 (2008).

[6] E. Y. Zhu, Q. Zhuang, and P. W. Shor, Superadditivity of the Classical Capacity with Limited Entanglement Assistance, Phys. Rev. Lett. 119, 040503 (2017).

[7] E. Y. Zhu, Q. Zhuang, M.-H. Hsieh, and P. W. Shor, Superadditivity in trade-off capacities of quantum channels, IEEE Trans. Inf. Theory 65, 3973 (2018).

[8] F. Leditzky, D. Leung, and G. Smith, Dephrasure Channel and Superadditivity of Coherent Information, Phys. Rev. Lett. 121, 160501 (2018).

[9] M. Fanizza, F. Kianvash, and V. Giovannetti, Quantum Flags and New Bounds on the Quantum Capacity of the Depolarizing Channel, Phys. Rev. Lett. 125, 020503 (2020).

[10] C. H. Bennett and S. J. Wiesner, Communication via Oneand Two-Particle Operators on Einstein-Podolsky-Rosen States, Phys. Rev. Lett. 69, 2881 (1992).

[11] C. H. Bennett, P. W. Shor, J. A. Smolin, and A. V. Thapliyal, Entanglement-Assisted Classical Capacity of Noisy Quantum Channels, Phys. Rev. Lett. 83, 3081 (1999).

[12] C. H. Bennett, P. W. Shor, J. A. Smolin, and A. V. Thapliyal, Entanglement-assisted capacity of a quantum channel and the reverse Shannon theorem, IEEE Trans. Inf. Theory 48, 2637 (2002).

[13] A. S. Holevo, On entanglement-assisted classical capacity, J. Math. Phys. (N.Y.) 43, 4326 (2002).

[14] P. W. Shor, The classical capacity achievable by a quantum channel assisted by limited entanglement, arXiv:quant-ph/ 0402129.

[15] M.-H. Hsieh, I. Devetak, and A. Winter, Entanglementassisted capacity of quantum multiple-access channels, IEEE Trans. Inf. Theory 54, 3078 (2008).

[16] Q. Zhuang, E. Y. Zhu, and P. W. Shor, Additive Classical Capacity of Quantum Channels Assisted by Noisy Entanglement, Phys. Rev. Lett. 118, 200503 (2017).

[17] M. M. Wilde and M.-H. Hsieh, The quantum dynamic capacity formula of a quantum channel, Quantum Inf. Process. 11, 1431 (2012).

[18] M. M. Wilde, P. Hayden, and S. Guha, Information TradeOffs for Optical Quantum Communication, Phys. Rev. Lett. 108, 140501 (2012).

[19] S. Lloyd, Capacity of the noisy quantum channel, Phys. Rev. A 55, 1613 (1997). 
[20] P. W. Shor, in Proceedings of the MSRI Workshop in Quantum Computation, Berkeley, 2002 (Mathematical Sciences Research Institute, Berkeley, 2002).

[21] I. Devetak, The private classical capacity and quantum capacity of a quantum channel, IEEE Trans. Inf. Theory 51, 44 (2005).

[22] V. Giovannetti, R. Garcia-Patron, N. J. Cerf, and A. S. Holevo, Ultimate classical communication rates of quantum optical channels, Nat. Photonics 8, 796 (2014).

[23] M. Rosati, A. Mari, and V. Giovannetti, Narrow bounds for the quantum capacity of thermal attenuators, Nat. Commun. 9, 4339 (2018).

[24] K. Sharma, M. M. Wilde, S. Adhikari, and M. Takeoka, Bounding the energy-constrained quantum and private capacities of phase-insensitive bosonic Gaussian channels, New J. Phys. 20, 063025 (2018).

[25] K. Noh, V. V. Albert, and L. Jiang, Quantum capacity bounds of Gaussian thermal loss channels and achievable rates with Gottesman-Kitaev-Preskill codes, IEEE Trans. Inf. Theory 65, 2563 (2018).

[26] K. Noh, S. Pirandola, and L. Jiang, Enhanced energyconstrained quantum communication over bosonic Gaussian channels, Nat. Commun. 11, 457 (2020).

[27] H. Shi, Z. Zhang, and Q. Zhuang, Practical Route to Entanglement-Assisted Communication over Noisy Bosonic Channels, Phys. Rev. Applied 13, 034029 (2020).

[28] S. Hao, H. Shi, W. Li, Q. Zhuang, and Z. Zhang, Entanglement-assisted communication surpassing the ultimate classical capacity, arXiv:2101.07482.

[29] M. E. Grein, M. L. Stevens, N. D. Hardy, and P. B. Dixon, Stabilization of long, deployed optical fiber links for quantum networks, in Conference on Lasers and ElectroOptics (Optical Society of America, San Jose, 2017), p. FTu4F.6.

[30] M. Fanizza, M. Rosati, M. Skotiniotis, J. Calsamiglia, and V. Giovannetti, Classical capacity of quantum Gaussian codes without a phase reference: When squeezing helps, arXiv:2006.06522.

[31] J. W. Goodman, Some fundamental properties of speckle, J. Opt. Soc. Am. 66, 1145 (1976).

[32] J. W. Goodman, Some effects of target-induced scintillation on optical radar performance, Proc. IEEE 53, 1688 (1965).

[33] Q. Zhuang, Z. Zhang, and J. H. Shapiro, Quantum illumination for enhanced detection of Rayleigh-fading targets, Phys. Rev. A 96, 020302(R) (2017).

[34] C. Weedbrook, S. Pirandola, R. García-Patrón, N. J. Cerf, T. C. Ralph, J. H. Shapiro, and S. Lloyd, Gaussian quantum information, Rev. Mod. Phys. 84, 621 (2012).

[35] M. Horodecki, P. W. Shor, and M. B. Ruskai, Entanglement breaking channels, Rev. Math. Phys. 15, 629 (2003).

[36] C. King, K. Matsumoto, M. Nathanson, and M. B. Ruskai, Properties of conjugate channels with applications to additivity and multiplicativity, Markov Process Relat. Fields 13, 391 (2007), http://math-mprf.org/journal/articles/id1123/.

[37] M.-H. Hsieh and M. M. Wilde, Entanglement-assisted communication of classical and quantum information, IEEE Trans. Inf. Theory 56, 4682 (2010).

[38] M. M. Wilde and M.-H. Hsieh, Public and private resource trade-offs for a quantum channel, Quantum Inf. Process. 11, 1465 (2012).
[39] K. Brádler, P. Hayden, D. Touchette, and M. M. Wilde, Trade-off capacities of the quantum Hadamard channels, Phys. Rev. A 81, 062312 (2010).

[40] C. H. Bennett, G. Brassard, C. Crépeau, R. Jozsa, A. Peres, and W. K. Wootters, Teleporting an Unknown Quantum State via Dual Classical and Einstein-Podolsky-Rosen Channels, Phys. Rev. Lett. 70, 1895 (1993).

[41] A. Anshu, R. Jain, and N. A. Warsi, Building blocks for communication over noisy quantum networks, IEEE Trans. Inf. Theory 65, 1287 (2019).

[42] H. Qi, Q. Wang, and M. M. Wilde, Applications of positionbased coding to classical communication over quantum channels, J. Phys. A 51, 444002 (2018).

[43] See Supplemental Material at http://link.aps.org/ supplemental/10.1103/PhysRevLett.126.060502 for details of the analyses and calculations, which includes Refs. [44-54].

[44] C. Adami and N. J. Cerf, Von Neumann capacity of noisy quantum channels, Phys. Rev. A 56, 3470 (1997).

[45] D. Elkouss and S. Strelchuk, Nonconvexity of private capacity and classical environment-assisted capacity of a quantum channel, Phys. Rev. A 94, 040301(R) (2016).

[46] A.S. Holevo, M. Sohma, and O. Hirota, Capacity of quantum Gaussian channels, Phys. Rev. A 59, 1820 (1999).

[47] M. M. Wolf, G. Giedke, and J. I. Cirac, Extremality of Gaussian Quantum States, Phys. Rev. Lett. 96, 080502 (2006).

[48] V. Giovannetti, A. Holevo, and R. A. García-Patrón, Solution of Gaussian optimizer conjecture for quantum channels, Commun. Math. Phys. 334, 1553 (2015).

[49] V. Giovannetti, A. S. Holevo, and A. Mari, Majorization and additivity for multimode bosonic Gaussian channels, Theor. Math. Phys. 182, 284 (2015).

[50] N. Datta and T. C. Dorlas, The coding theorem for a class of quantum channels with long-term memory, J. Phys. A 40, 8147 (2007).

[51] N. Datta and T. Dorlas, Classical capacity of quantum channels with general Markovian correlated noise, J. Stat. Phys. 134, 1173 (2009).

[52] H. Boche, G. Janßen, and S. Kaltenstadler, Entanglementassisted classical capacities of compound and arbitrarily varying quantum channels, Quantum Inf. Process. 16, 88 (2017).

[53] H. Boche, G. Janßen, and S. Kaltenstadler, Entanglement assisted classical capacity of compound quantum channels, in 2016 IEEE International Symposium on Information Theory (ISIT) (IEEE, New York, 2016), pp. 1680-1684.

[54] M. Berta, H. Gharibyan, and M. Walter, Entanglementassisted capacities of compound quantum channels, IEEE Trans. Inf. Theory 63, 3306 (2017).

[55] J. Shapiro, S. Guha, and B. Erkmen, Ultimate channel capacity of free-space optical communications, J. Opt. Commun. Netw. 4, 501 (2005).

[56] B. Sklar, Rayleigh fading channels in mobile digital communication systems. I. Characterization, IEEE Commun. Mag. 35, 90 (1997).

[57] A. Arqand, L. Memarzadeh, and S. Mancini, Quantum capacity of bosonic dephasing channel, Phys. Rev. A 102, 042413 (2020). 